\title{
Research Article \\ Effect of the Longitudinal Contact Location on Vehicle Dynamics Simulation
}

\author{
N. Burgelman, Z. Li, and R. Dollevoet \\ Faculty of Civil Engineering and Geosciences, Section of Road and Railway Engineering, Delft University of Technology, \\ Stevinweg 1, 2628 CN Delft, Netherlands
}

Correspondence should be addressed to Z. Li; z.li@tudelft.nl

Received 27 October 2015; Revised 31 December 2015; Accepted 10 January 2016

Academic Editor: Enrico Meli

Copyright (c) 2016 N. Burgelman et al. This is an open access article distributed under the Creative Commons Attribution License, which permits unrestricted use, distribution, and reproduction in any medium, provided the original work is properly cited.

This paper investigates the effect of the calculation of the longitudinal location of a wheel rail contact point on the wheelset's motion in a vehicle dynamic simulation. All current vehicle dynamic software programs assume that the contact between wheel and rail takes place in the vertical plane through the wheelset's rolling axis. However, when the yaw angle of the wheelset is nonzero, the contact point is situated up to $10 \mathrm{~mm}$ from that plane. This difference causes a difference in the yaw moment on the wheelset which is used in the vehicle dynamic simulation. To such an end, an existing analytical method to determine the longitudinal method was validated using a numerical approach. Then vehicle dynamic simulations with both the classic and the new contact location were performed, concluding that using a more accurate contact point location results in a smaller wheelset yaw angle in a vehicle dynamic simulation, although the effect is small.

\section{Introduction}

To assess the wheel rail contact conditions (e.g., at a turnout) a vehicle simulation and a contact method are needed. There are two possible approaches. Either one can use contact models online in the vehicle simulation, which are fast and robust, yet reasonably accurate. Or one can first use a simple method contact method to evaluate the contact forces during the vehicle simulation and then a sophisticated method offline to resolve the contact problems and obtain a more accurate calculation of the local stress and slip distribution in the contact area. In the latter case, the balance of forces is not necessarily fulfilled as the contact forces calculated with the sophisticated method are not coupled back to the vehicle dynamic simulation, therefore producing an error. The difference between the simple and the sophisticated contact method can be in the calculated contact forces or in the calculated contact location. This paper will focus on the effect of the contact location, whereas the effect of the calculation of the tangential contact force is covered by [1].

A common assumption in vehicle dynamics simulations is that the contact between the wheel and the rail takes place in the vertical plane through the rolling axis of the wheelset. This is correct when there is no angle of attack; however, when the angle of attack is not zero, the actual contact point will shift in longitudinal direction, opposite to the direction of the yaw rotation of the wheelset; see Figure 1. Therefore the yaw moment caused by the lateral force of the rail on the wheel will be smaller than expected; that is, not accounting for this longitudinal shift in the contact point would lead to an overestimation of this yaw moment. As this moment acts to increase the yaw angle, an overestimation of this moment will lead to an overestimation of the angle of attack of the wheelset, which in turn would give an overestimation of the derailment risk, as some derailment criteria are based on the angle of attack (e.g., $[2,3])$.

In this paper, the effect of the longitudinal location of the contact point on the wheelset's yaw angle is quantified through vehicle dynamic simulations. Therefore a method is needed to calculate the longitudinal location of the contact point. We propose to use the principle of contact locus, a line of potential contact points on the wheel profile (see Section 2.1). In this way, we avoid the need for a true 3D method such as that found in $[4,5]$. When the angle of attack is zero the contact locus is the principal wheel profile. 


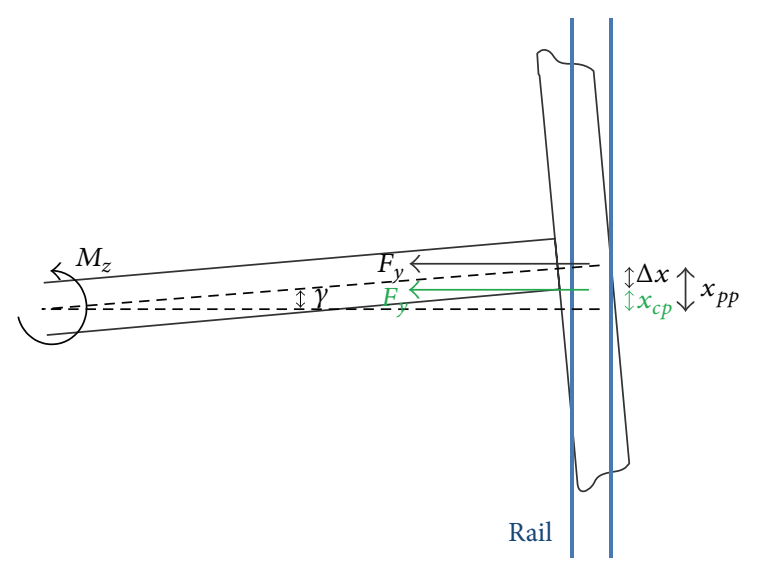

FIgURE 1: A top view of a half wheelset with an angle of attack, $\gamma$. The lateral force of the wheel on the rail occurs at the longitudinal contact location defined $\left(x_{c p}\right)$, whereas current vehicle dynamic simulations assume that the contact occurs at the principal profile (longitudinal location defined by $x_{p p}$ ).

However, when the angle of attack is not zero, a method is needed to calculate the contact locus. Here, we use an analytical approximation: Wang's method [6] (translated as Appendix D of Li's thesis [7]), based on work of de Pater [8, 9], explained in Section 2.2. This method is fast and so suitable for online evaluation during the vehicle dynamic simulation. Wang's method is compared to results obtained from the projection of a 3D mesh, explained in Section 2.3. Due to the interpolations and the numerical minimum search required, the $3 \mathrm{D}$ meshing method is considered too slow to be used in vehicle dynamics simulation. However, the accuracy of the 3D meshing method only depends on the mesh size; therefore it allows validating Wang's method.

\section{Determination of the Contact Location}

2.1. Contact Locus. To determine the contact point we assume that the rail is straight and smooth in the longitudinal (rolling) direction, whereas in the lateral direction (i.e., along the rail cross section) we still allow rail surface irregularities. This assumption is widely used in vehicle dynamic simulation, although violated in the case of wheel rail contact at the point of a frog or at the gap of an insulated joint. If these are the areas of interest, it is better to use a finite element simulation instead, for example, Oregui et al. [10] for FEM of contact at rail joints or Zhao and Li [11] and Telliskivi and Olofsson [12] for general wheel/rail contact. However, due to the large computational effort, finite element methods cannot replace simpler contact methods for the evaluation of the contact forces in a vehicle dynamics simulation.

The assumption of straight and smooth rail allows simplifying the problem by introducing the concept of "contact locus." A contact locus is a line of points on the wheel surface where contact is possible, given a certain angle of attack and roll angle of the wheelset. A contact locus can be used instead of a wheel profile, so that instead of searching for contact

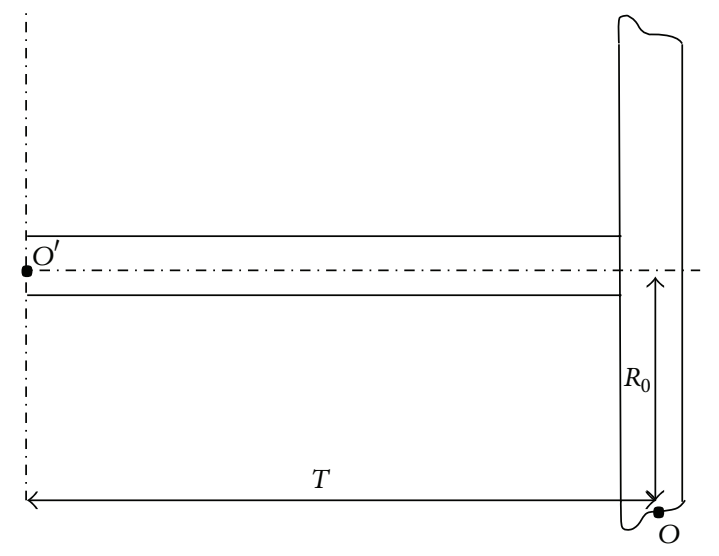

FIGURE 2: Side view of a half wheelset with the reference frame of the wheel profile $(O)$ and the reference frame at the center of the wheelset $\left(O^{\prime}\right)$ with $T$, half the tape circle distance, and $R_{0}$, the rolling radius.

between the wheel and the rail surfaces one can search for contact between the contact locus and the rail profile, so that the contact point search problem is reduced to being in the $2 \mathrm{D}$ domain.

2.2. Wang's Method. Wang's method is an analytical approximation; it calculates a correction of the wheel rotated principal profile, that is, the wheel profile in a vertical plane through the axle of the wheelset. Principal wheel profiles are commonly defined as a list of points $\left(y_{O}(i), z_{O}(i)\right)$, where $y$ is the lateral and $z$ the vertical direction, and with the origin in $O$, which is located at half the tape circle distance $(T)$ away from the wheelset's center $\left(O^{\prime}\right)$ in lateral direction and at vertical distance $R_{0}$ from the wheel axis; see Figure 2. This way the local rolling radius in each point on the wheel profile is $R(i)=R_{0}-z(i)$. The rotated wheel principal profiles $\left(x_{p p}(i)\right.$, $\left.y_{p p}(i), z_{p p}(i)\right)$ are the principal profile $\left(y_{O}(i), z_{O}(i)\right)$ rotated around the centre of the wheelset $\left(O^{\prime}\right)$ with the angle of attack and the roll angle of the wheelset. The wheel principal rotated profile can be calculated by translating the reference frame in which the wheel profile is defined to the center of the wheelset and then rotate it around the yaw angle (= angle of attack) $(\gamma)$ and the roll angle $(\theta)$ :

$$
\begin{aligned}
& {\left[\begin{array}{l}
x_{p p}(i) \\
y_{p p}(i) \\
z_{p p}(i)
\end{array}\right]} \\
& \quad=\left[\begin{array}{ccc}
\cos \gamma & \sin \gamma & 0 \\
-\sin \gamma & \cos \gamma \cos \theta & \sin \theta \\
0 & -\sin \theta & \cos \theta
\end{array}\right]\left[\begin{array}{c}
0 \\
y_{0}(i)+T \\
z_{0}(i)-R_{0}
\end{array}\right] .
\end{aligned}
$$

Wang's method assumes that at each point on the wheel profile the wheel surface can be approximated as a cone [6]. These cones pass through a point on the wheel profile, with the axes coinciding with the wheelset's rolling axis and a cone angle equal to the local contact angle. According to Wang 
[6], the contact locus $\left(x_{c p}(i), y_{c p}(i), z_{c p}(i)\right)$ is then found as a correction $(\Delta x(i), \Delta y(i), \Delta z(i))$ of the rotated principal profiles as follows [6]:

$$
\begin{aligned}
\Delta x(i)= & x_{c p}(i)-x_{p p}(i) \\
= & R(i) \sin \theta \sin \gamma+l_{x} R(i) \tan \delta(i), \\
\Delta y(i)= & y_{c p}(i)-y_{p p}(i) \\
= & R(i) \sin \theta \cos \gamma \\
& -\frac{R(i)}{1-l_{x}^{2}}\left(l_{x}^{2} l_{y} \tan \delta(i)+l_{z} m\right), \\
\Delta z(i)= & z_{c p}(i)-z_{p p}(i) \\
= & R(i) \cos \theta-\frac{R(i)}{1-l_{x}^{2}}\left(l_{x}^{2} l_{y} \tan \delta(i)-l_{y} m\right),
\end{aligned}
$$

where $\delta(i)$ is the local angle of the wheel surface with respect to the wheelset rolling axis:

$$
\begin{aligned}
& l_{x}=\cos \theta \sin \gamma, \\
& l_{y}=\cos \theta \cos \gamma, \\
& l_{z}=\sin \theta, \\
& m=\sqrt{1-l_{x}^{2}\left(1+\tan ^{2} \delta(i)\right)} .
\end{aligned}
$$

For the derivation of (2)-(3), including the physical meaning of the parameters in (3), we refer to Wang [6] or the English translation in the appendix of Li's thesis [7].

2.3. Spline Interpolation on a 3D Mesh. To validate Wang's method, a 3D mesh of the wheel surface has been constructed. A matrix of points on the wheel surface is obtained by revolving the wheel profile around the wheelset's rolling axis, using the parametric equation for a circle through each point on the wheel profile. All the points are then rotated using (1); then a $3 \mathrm{D}$ cubic spline surface is fitted to the mesh points so that the resulting surface is the rotated wheel surface. On the intersection line of this spline surface and a plane vertical and parallel to the rails (semitransparent purple plane in Figure 3), the minimum of $z$ is searched, for a given lateral location, using a golden section search. The resulting $z$ and the corresponding $x$ are the coordinates of the contact locus. The surface as well as the contact locus (in red) and the rotated principal profile (blue) are shown in Figure 3 for an angle of attack of $5^{\circ}$. This is done for a better visualization as common angles of attack are $0^{\circ}$ to $0.5^{\circ}$ for trains and $0^{\circ}$ to $2^{\circ}$ for trams. The advantage of the meshing method is that it does not make geometric simplifications; thus the accuracy only depends on the mesh size, which can always be refined. The disadvantage is that the minimum search requires a substantial computational effort; therefore the method is not suitable for evaluating the contact point location online in a vehicle dynamic simulation.

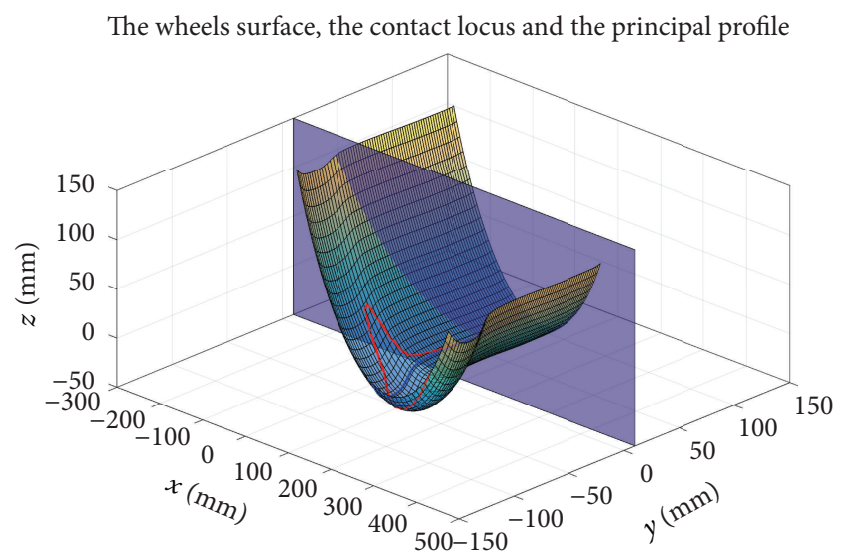

FIgURE 3: The 3D mesh with the contact locus as a red line and the principal profile as a blue line, and the plane in which the minimum is to be found as a semitransparent purple surface. For visualization it is calculated here with a very large angle of attack of $5^{\circ}$ and a coarse mesh.

\section{Results}

3.1. Comparison of Methods for Contact Locus. Comparing the results obtained from Wang's method with the results from the 3D mesh for an angle of attack of $1^{\circ}$ shows a very good fit; see Figures 4 and 5. It is therefore concluded that Wang's method is sufficiently accurate for use in vehicle dynamic simulations.

Looking at Figure 5, one can see that the largest longitudinal difference between the rotated principal profile and the real contact locus is around $23 \mathrm{~mm}$. However, this largest difference occurs at the highest contact angle $\left(70^{\circ}\right)$, which will normally not be reached in a common vehicle dynamic simulation.

3.2. Effect on Vehicle Dynamics. Current vehicle dynamic simulations assume the contact takes place on the principal profile at $\left(x_{p p}, y_{p p}, z_{p p}\right)$, whereas it takes place at $\left(x_{c p}, y_{c p}\right.$, $\left.z_{c p}\right)$, calculated in the previous section. The location of the contact point has an effect on the moment on the wheelset transferred from the rail to the wheel (see Figure 1). The moment from the lateral wheel/rail contact force on the wheelset is normally calculated as $M_{z}=F_{y} x_{p p}$, whereas it should be $M_{z}=F_{y} x_{c p}$, where $F_{y}$ is the lateral force from the wheel on the rail consisting of the lateral components of the normal contact force and the lateral creep force.

A cosimulation was set up between the MultiBody Software (MBS) VIRail and Matlab in a similar way as presented in [1]. At each timestep of the simulation the angle of attack, the roll angle, the lateral creep force, the normal contact force, and the contact angle are passed from the MBS to Matlab. Matlab then calculates the lateral force and the difference in moment $\left(\Delta M_{z}=\Delta x F_{x}\right)$ for each wheel. The resulting moment is then fed back to the MBS where it is applied through an actuator.

The differences in longitudinal location and the resulting moment for a case of a vehicle entering a curve with radius 


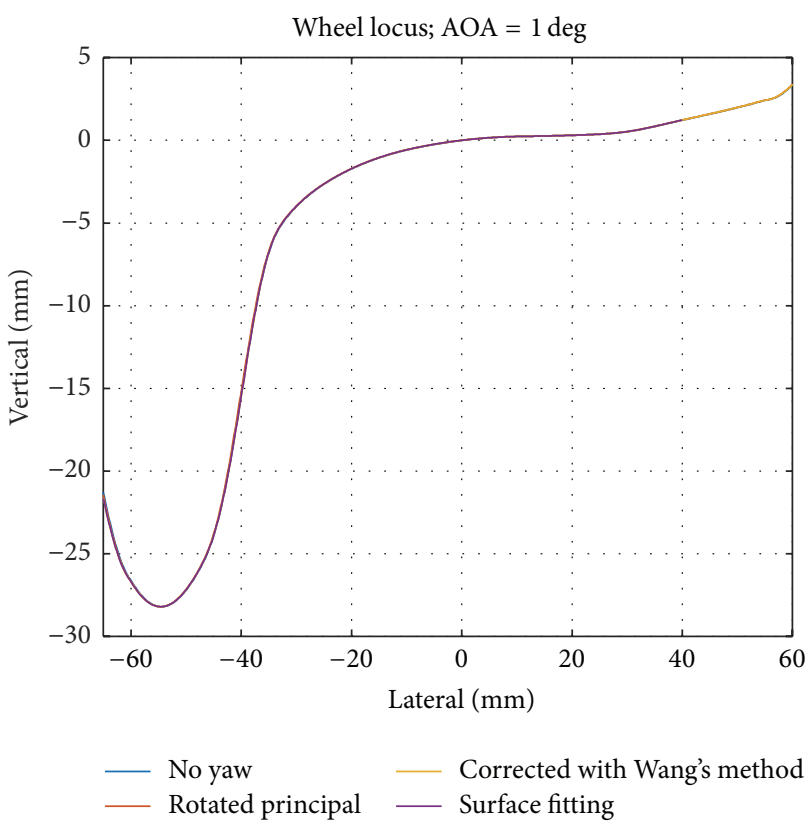

(a)

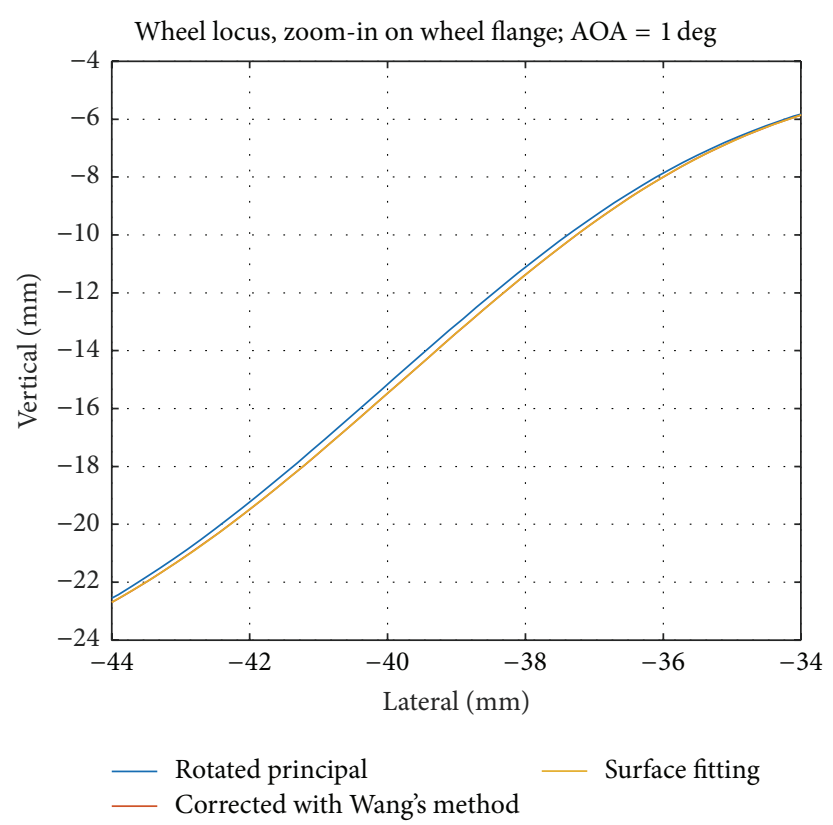

(b)

Figure 4: The projection of the contact locus on the $y z$-plane, vertical and perpendicular to the rail, for a s1002 profile and an angle of attack of $1^{\circ}$. The whole profile (a) and a zoom-in on the wheel flange (b).

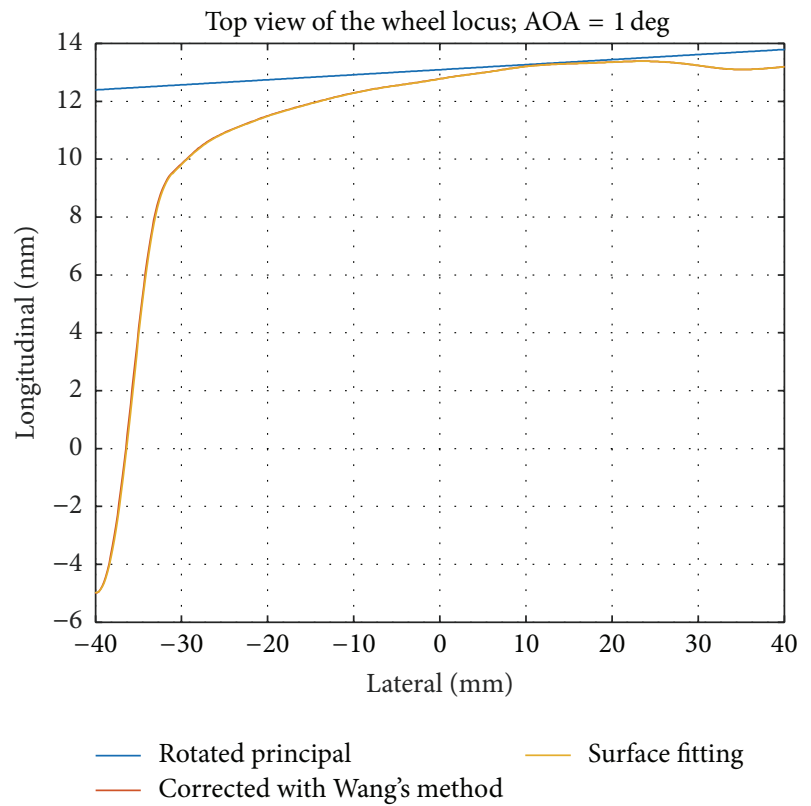

Figure 5: The projection of the contact locus on the $y x$-plane (horizontal plane), for a s1002 profile and an angle of attack of $1^{\circ}$.

$100 \mathrm{~m}$ (realistic for tram networks) are plotted in Figures 6(a) and 6(b). This moment results in a change in the angle of attack in the simulation, seen in Figure 7. It can be seen that the influence of this moment on the angle of attack is very small at around $0.2 \%$. This can be explained by the observation that the moment applied through the actuator is small compared to the other yaw moments that act on the wheelset, more specifically, the yaw moment caused by the longitudinal creep force. The longitudinal creep force causes a yaw moment equaling $F_{x} T$, where $T$ is half the tape circle distance, and even though $F_{x}$ is generally smaller than $F_{y}, \Delta x$ is much smaller than $T$, so that the moment originating from the lateral contact force becomes negligible.

We conclude that, for the case of a vehicle entering a $100 \mathrm{~m}$ curve, resulting in an angle of attack of $1^{\circ}$ and a contact angle of $40^{\circ}$, the influence of the longitudinal contact location is negligible. In tramway the angle of attack can be as high as $2^{\circ}$, and in case of a derailment the contact angle can be up to $70^{\circ}$. However, such angles of attack will only occur in very tight curves so that the difference in rolling radius between the left and right wheel will not compensate for the difference in traveled distance on the left and right rail; therefore in such cases there will always be a high longitudinal creep force so that the change in moment due to the longitudinal contact location can safely be neglected.

\section{Conclusions}

The exact longitudinal location of the contact point can be calculated for normal wheel/rail contact fast and accurately using Wang's method, which was validated using a 3D mesh. The contact location calculated with Wang's method was used in a vehicle dynamic simulation. The results from this simulation were compared with results where the contact location was calculated in the classical way. It was concluded that more accurate contact location from Wang's method results in a smaller angle of attack.

However, even though the extra computational time for obtaining such accurate longitudinal contact location is 


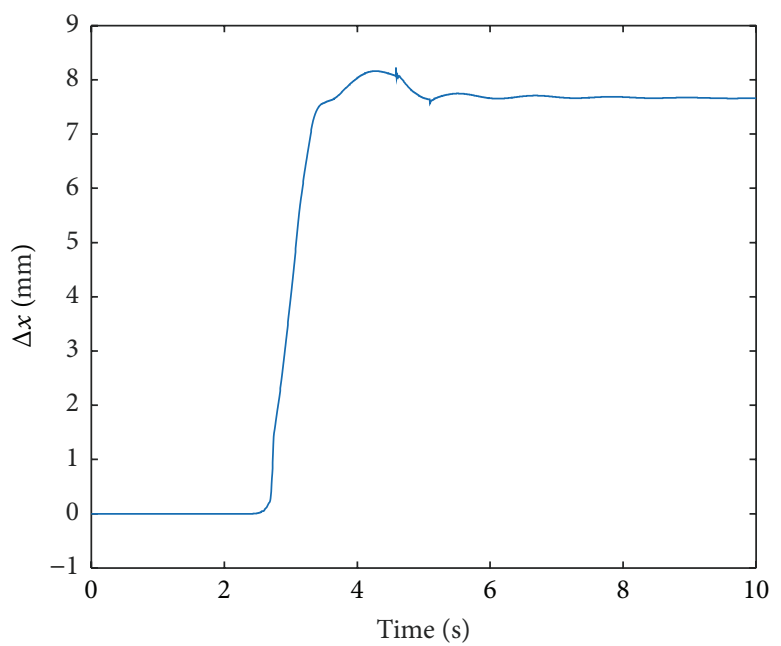

(a)

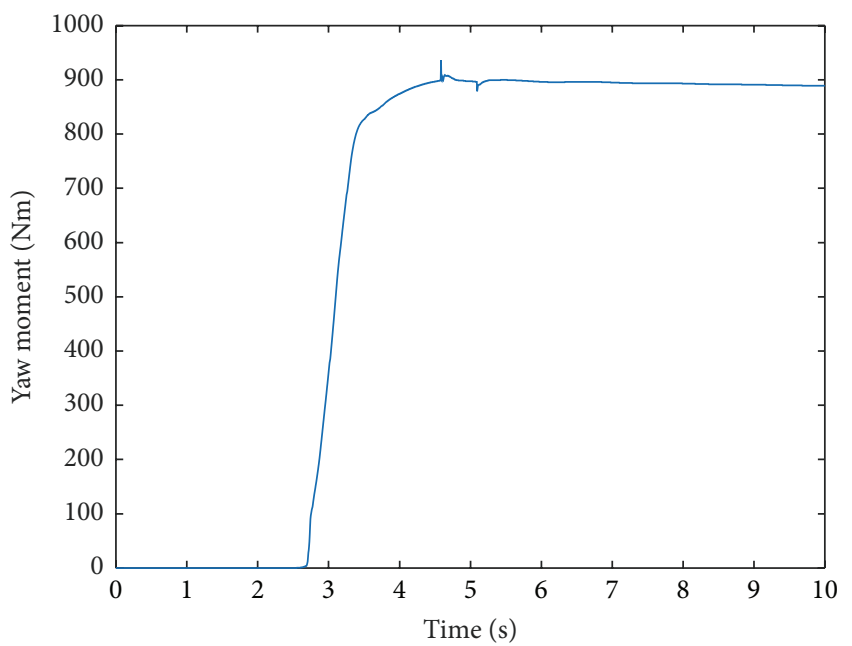

(b)

FIGURE 6: The longitudinal distance between the contact locus obtained with Wang's method and the principal profile at the point of contact (a), and the difference in yaw moment due to the assumed location of the contact patch (b). This difference in moment is applied through the actuator in the MBS.

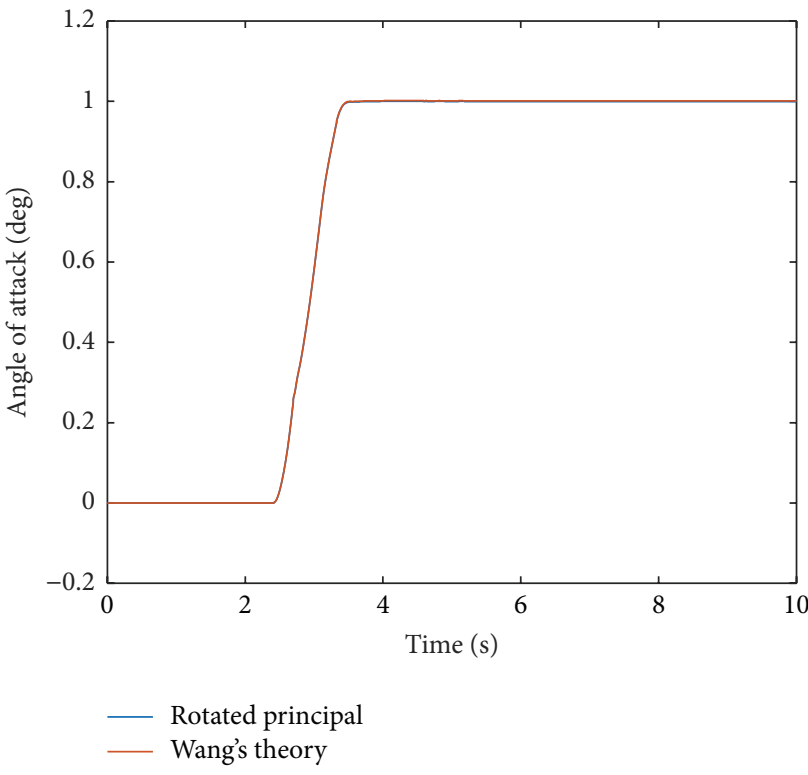

(a)

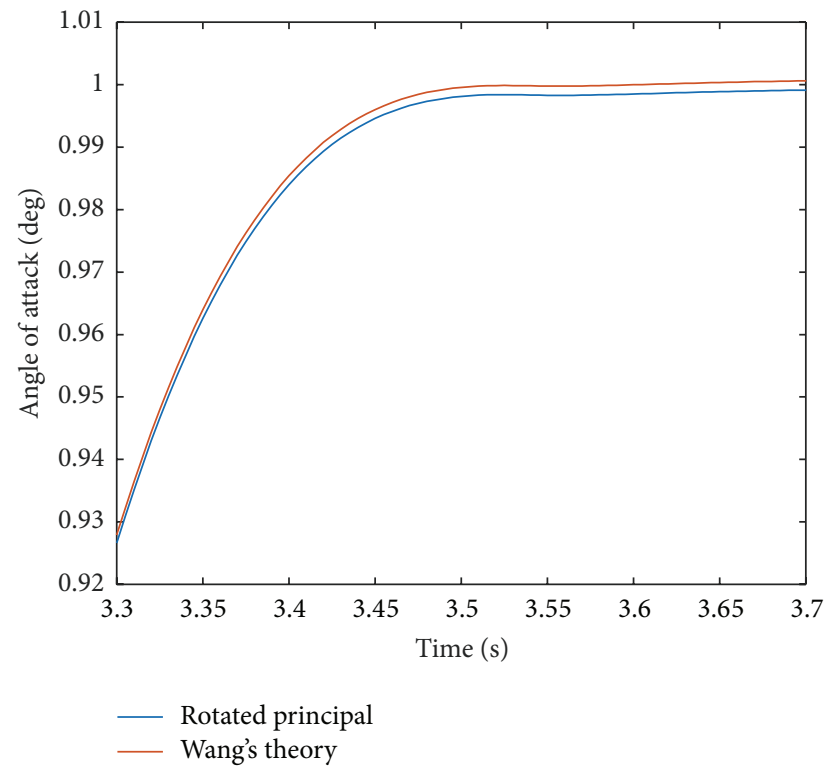

(b)

Figure 7: The angle of attack of the first wheelset of a vehicle entering a curve with radius $100 \mathrm{~m}$ from a simulation with the contact on the rotated principal profile and for a simulation with the contact location obtained through Wang's method (a) and a zoom-in on the moment steady curving is reached $(\mathrm{b})$.

small, also the benefits are small, as simulations have shown that the effect of the longitudinal location of the contact point on the vehicle simulation is negligible for contact angles up to $40^{\circ}$ and angles of attack up to $1^{\circ}$; for derailment scenarios with contact angles up to $70^{\circ}$ further investigation is necessary.

\section{Conflict of Interests}

The authors declare that there is no conflict of interests regarding the publication of this paper.

\section{References}

[1] N. Burgelman, M. S. Sichani, R. Enblom, M. Berg, Z. Li, and R. Dollevoet, "Influence of wheel-rail contact modelling on vehicle dynamic simulation," Vehicle System Dynamics, vol. 5, pp. 11901203, 2015.

[2] J. Elkins and H. M. Wu, "Angle of attack and distance based criterion for flange climb derailment," Vehicle System Dynamics, vol. 33, pp. 293-305, 1999.

[3] F. Braghin, S. Bruni, and G. Diana, "Experimental and numerical investigation on the derailment of a railway wheelset with 
solid axle," Vehicle System Dynamics, vol. 44, no. 4, pp. 305-325, 2006.

[4] A. Pieringer, W. Kropp, and J. C. O. Nielsen, "The influence of contact modelling on simulated wheel/rail interaction due to wheel flats," Wear, vol. 314, no. 1-2, pp. 273-281, 2014.

[5] M. Malvezzi, E. Meli, S. Falomi, and A. Rindi, "Determination of wheel-rail contact points with semianalytic methods," Multibody System Dynamics, vol. 20, no. 4, pp. 327-358, 2008.

[6] K. Wang, "The track of wheel contact points and the calculation of wheel/rail geometric contact parameters," Journal of Southwest Jiaotong University, vol. 1, pp. 89-99, 1984.

[7] Z. Li, Wheel-rail rolling contact and its application to wear simulation [Ph.D. thesis], Delft University of Technology, Delft, The Netherlands, 2002.

[8] A. D. de Pater, "The general theory of the motion of a single wheelset moving through a curve with constant radius and cant," Journal of Applied Mathematics and Mechanics, vol. 61, no. 7, pp. 277-292, 1981.

[9] A. D. de Pater, "The geometrical contact between track and wheelset," Vehicle System Dynamics, vol. 17, no. 3, pp. 127-140, 1988.

[10] M. Oregui, Z. Li, and R. Dollevoet, "An investigation into the relation between wheel/rail contact and bolt tightness of rail joints using a dynamic finite element model," in Proceedings of the 9th International Conference on Contact Mechanics and Wear of Rail/Wheel Systems (CM '12), pp. 501-508, Chengdu, China, August 2012.

[11] X. Zhao and Z. Li, "The solution of frictional wheel-rail rolling contact with a $3 \mathrm{D}$ transient finite element model: validation and error analysis," Wear, vol. 271, no. 1-2, pp. 444-452, 2011.

[12] T. Telliskivi and U. Olofsson, "Contact mechanics analysis of measured wheel-rail profiles using the finite element methods," Journal of Rail Transportation, vol. 215, pp. 65-72, 2001. 


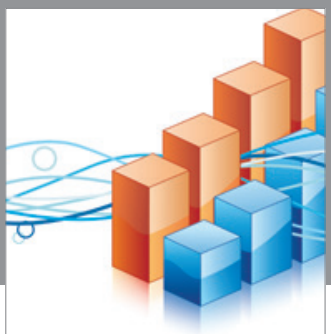

Advances in

Operations Research

vatem alat4

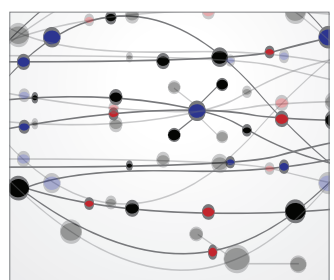

\section{The Scientific} World Journal
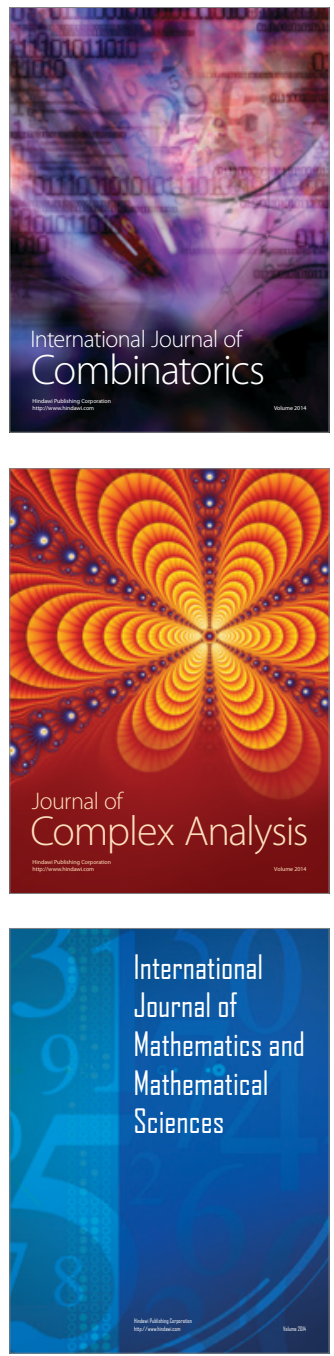
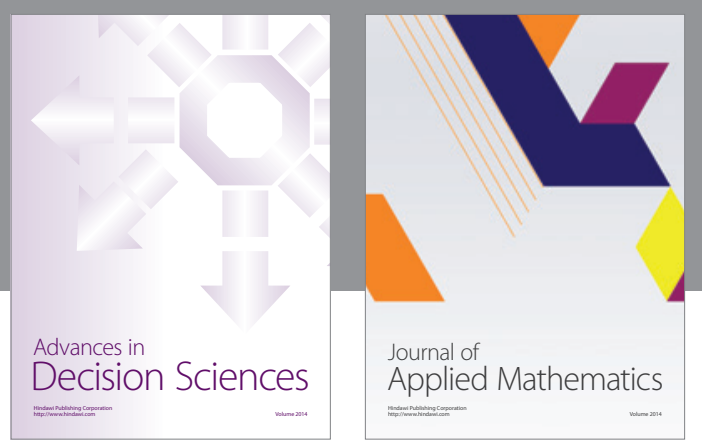

Algebra

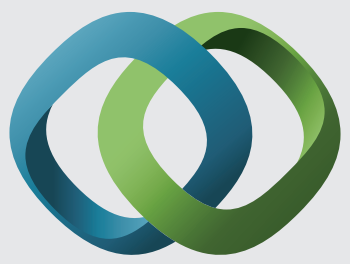

\section{Hindawi}

Submit your manuscripts at

http://www.hindawi.com
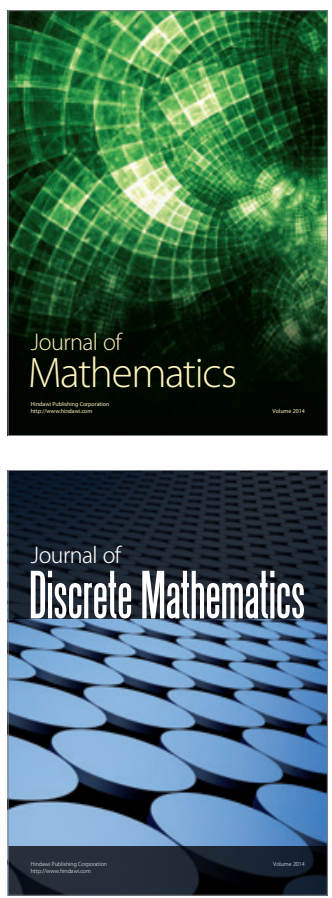

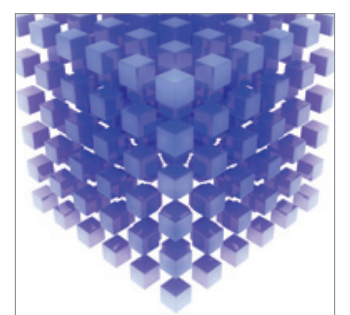

Mathematical Problems in Engineering
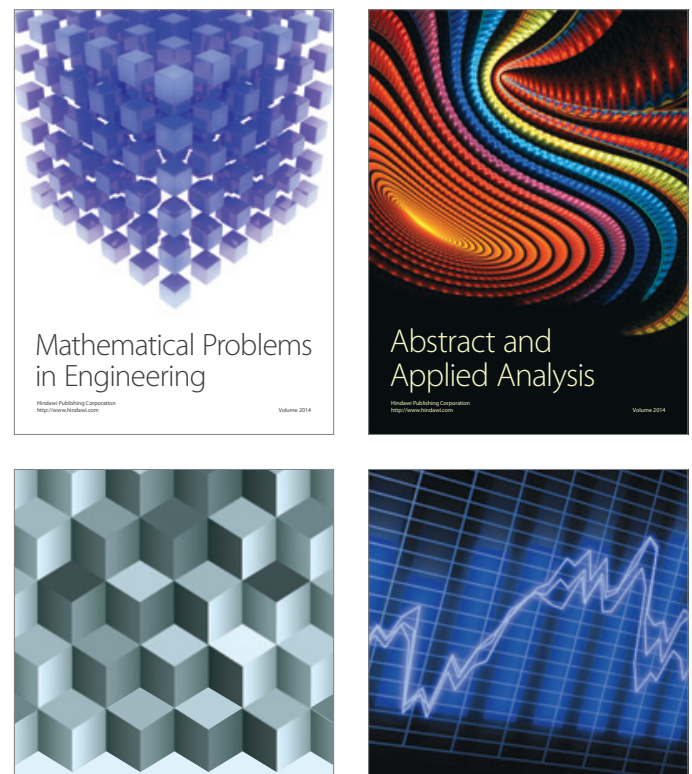

Journal of

Function Spaces

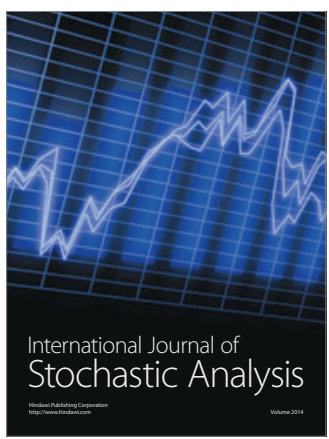

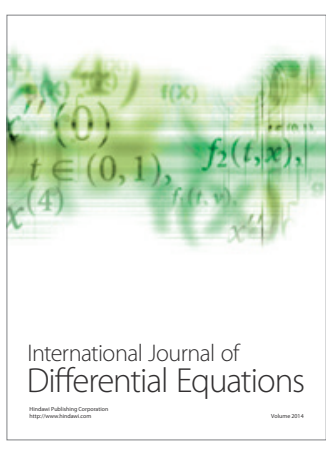
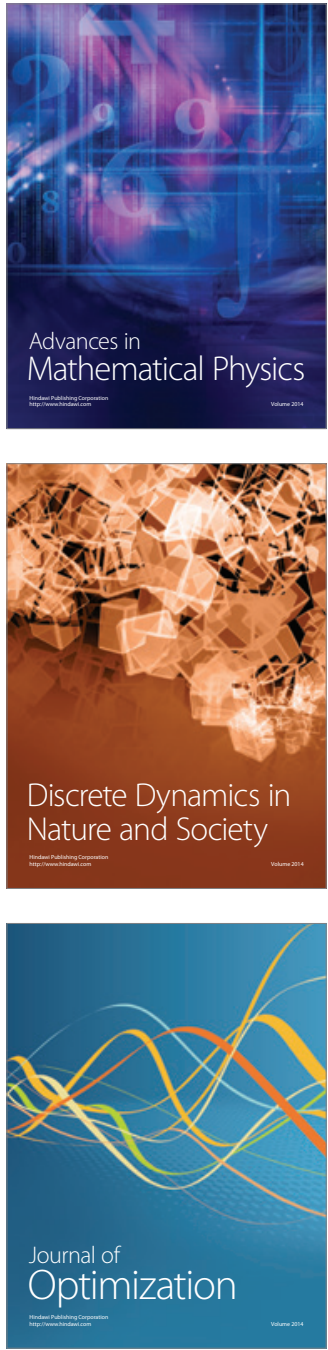